\title{
A surprising finding after adenosine administration
}

\author{
C. Timmermans ${ }^{1} \cdot \mathbf{H}$. Wellens ${ }^{2}$
}

Published online: 26 April 2017

(C) The Author(s) 2017. This article is an open access publication.

\begin{abstract}
Answer
To facilitate the explanation of Fig. 1, the QRS complexes are labelled. The first two show a normal QRS preceded by a normal PR interval. Thereafter the QRS configuration changes. Complexes 3 to 6 show gradual widening, a frontal axis shift to horizontal and loss of the initial $\mathrm{q}$ in leads I and V6, an rS pattern in lead III, and the development of a left bundle branch block-like QRS configuration. There is also a gradual lengthening of the PR interval. These adenosineinduced changes can be explained by progressive delay and block in AV nodal conduction and progressive activation of the ventricle over a right-sided atriofascicular $(\mathrm{AF})$ fibre, also known as a Mahaim fibre. This structure inserts in the ventricle in or close to the right bundle branch, ultimately resulting in the typical QRS configuration of beats 5 and 6 [1]. Then $4 \mathrm{P}$ waves are not conducted to the ventricle because of complete block both in the AV node and the AF fibre. As described several years ago, in contrast to rapidly conducting accessory pathways, the AF fibre is sensitive to adenosine resulting in delay and block [2]. It is explained by its AV nodal-like cellular composition. In our patient, after adenosine administration during sinus rhythm, complete AV nodal block preceded block in the AF fibre. A similar observation was reported by Belhassen et al. [3]. Of interest is also what happened after the episode of complete AV block. QRS 7 shows AV nodal conduction. QRS 8 is
\end{abstract}

C. Timmermans

ccmm.timmermans@mumc.nl

1 Department of Cardiology, Maastricht University Medical Center, Maastricht, The Netherlands

221 Henric van Veldekeplein, Maastricht, The Netherlands a paced beat from the apex of the right ventricle, with QRS 9 showing anterograde $\mathrm{AV}$ conduction only over the AF fibre, indicating retrograde invasion into the normal conduction system. QRS 10 shows AV nodal conduction again with a small contribution to ventricular activation over the $\mathrm{AF}$ fibre. QRS 11 is similar to QRS 1 and 2, and has a normal $\mathrm{PR}$, suggesting complete ventricular activation over the AV node. The recording in Fig. 1 clearly shows the short-lasting effect of intravenous adenosine on conduction over the two $\mathrm{AV}$ connections. In the patient, tachycardias with anterograde AV conduction over the AF fibre could be initiated by appropriately timed atrial premature beats. Conduction over the AF fibre was terminated by catheter ablation.

Conflict of interest C. Timmermans and H. Wellens declare that they have no competing interests.

Open Access This article is distributed under the terms of the Creative Commons Attribution 4.0 International License (http:// creativecommons.org/licenses/by/4.0/), which permits unrestricted use, distribution, and reproduction in any medium, provided you give appropriate credit to the original author(s) and the source, provide a link to the Creative Commons license, and indicate if changes were made.

\section{References}

1. Sternick EB, Timmermans C, Sousa E, et al. The electrocardiogram during sinus rhythm and tachycardia in patients with anterograde conduction over Mahaim fibers. The importance of an ' $r S$ ' pattern in lead III. J Am Coll Cardiol. 2004;44:1625-35.

2. Ellenbogen KA, Rogers R, Old W. Pharmacological characterization of conduction over a Mahaim fiber: evidence of adenosine sensitive conduction. Pacing Clin Electrophysiol. 1989;12:1396-402.

3. Belhassen B, Glick A. QRS complexes with a left bundle branch block morphology during adenosine triphosphate test: What is the diagnosis? J Cardiovasc Electrophysiol. 2002;13:519-20. 
Adenosine I.V. $21 \mathrm{mg}$

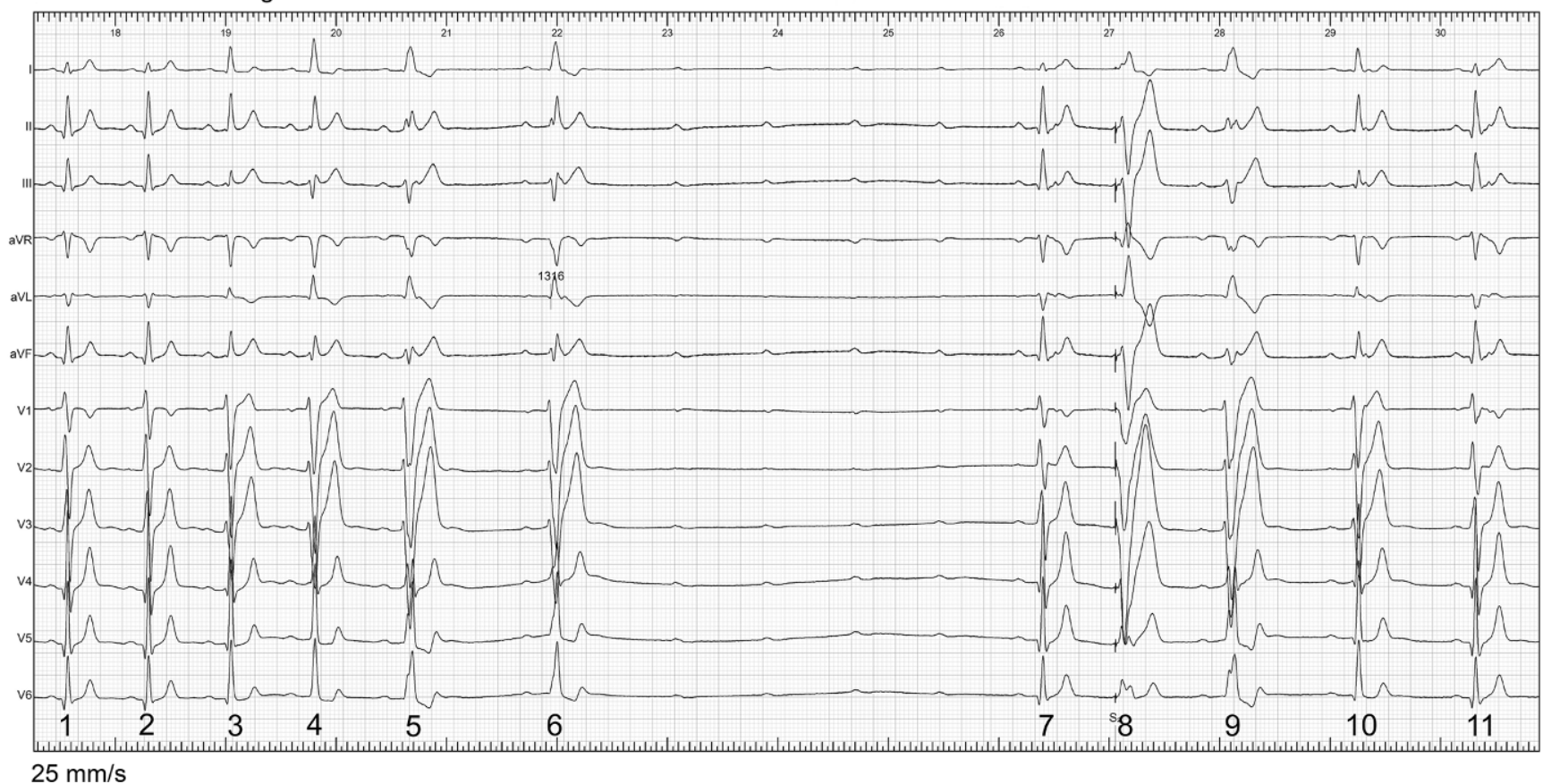

Fig. 1 Twelve-lead ECG after adenosine administration during sinus rhythm 